\title{
测绘工程中 GIS 数据的建库思路
}

\section{Ideas of Building GIS Database in Surveying and Mapping Engineering}

王长江

\author{
Changjiang Wang
}

河北省地矿局第六地质大队

中国·河北 石家庄 050000

Sixth Geological Brigade of Hebei Geology and Mineral Resources Bureau,

Shijiazhuang, Hebei, 050000, China
【摘要】针对测绘工程中 GIS 数据的建库进行研究, 阐述了 GIS 数据建库的主要技术思 路, 提出了数据库建模标准, 以及原始数据的采集、整理、检验以及入库等流程规范, 希望 对相关从业人员提供参考与借鉴。

【Abstract】This paper studies the construction of GIS database in surveying and mapping engineering, expounds the main technical ideas of GIS database construction, and puts forward the database modeling standards, as well as the original data collection, sorting, inspection and warehousing process specifications, hoping to provide reference and reference for relevant practitioners.

【关键词】地质测绘; GIS; 数据库; 技术思路

【Keywords \geological mapping; GIS; database; technical ideas

【DOI】10.36012/se.v2i2.1540

\section{1 引言}

地理信息数据库是测绘工程的重要组成部分, GIS 数据 库的完善性会直接影响到地质测绘工作的质量与效率。因此, 开展 GIS 数据库对测绘工作具有重要意义。基于此, 本文针对 建库整体流程进行研究分析, 同时从本质环节入手找出影响 建库的主要因素,并且制定出科学合理的建库技术方案,有效 落实 GIS 数据库的建设工作。

\section{GIS 数据建库主要技术思路}

\section{1 建库技术思路}

GIS 数据建库时要选用科学的工程管理技术, 同时对数 据库的标准化问题及数据信息准确性问题进行管控。建库流 程主要包括 CAD 数据处理、制定数据格式、添加相关要素属 性,保证数据信息准确是 GIS 数据建库中的重要指标, 在每个 环节都要围绕数据处理效果进行严格检查, 以此来对数据信 息的准确度与完整性提供保障[1]。

\section{2 建库基本流程}

在进行 GIS 数据建库时, 应该按照以下流程执行作业: (1) 数据库建模, 根据工程项目的测绘要求制定合适的数据库规 范, 同时制定相应的建库标准, 从而满足测绘工程的各项应用 需求; (2)采集整体数据, 保证基础数据的完备性是 GIS 建库工 作的基础, 技术团队要在正式开展测绘前掌握各项关键技术, 并且要对基础数据的准确性进行核对; 3)整理原始数据,按照
GIS 数据库规范要求对数据进行整理分析, 并且根据数据的 图层、类别以及属性进行区分, 制作表格; (4)检验数据, 针对数 据库与实际工程项目的匹配程度进行检验，同时对数据信息 质量进行检测; (5)数据库导入环节, 准确将各类数据信息导入 数据库中, 以此完成 GIS 数据的建库工作。

\section{GIS 数据建库整体流程}

\section{1 数据库建模}

GIS 数据建库的关键在于制定的数据库标准规范可以满 足工程项目要求。同时准确严格的数据库建库规范也能够约 束操作人员的工作态度, 使其采用科学的技术手段对各类基 础数据进行采集与整理, 如果在基础环节无法保证数据信息 的准确度, 便会导致建库失败 ${ }^{[2]}$ 。因此, GIS 数据库建模应该对 数据建库标准与规范进行综合考虑, 同时, 结合实际应用进行 权衡取舍。表 1 为河北省地矿局第六地质大队某测绘工程建 模实例。

\section{2 采集原始数据}

采集原始数据是 GIS 数据建库的重要环节, 在执行过程 中要围绕现场实际情况选择技术方式, 例如, 图解法与解析法 等。将数据库实际需求作为基础, 从以下两点展开: (1)针对地 理原始数据进行采集, 采集环节紧密贴合数据库建库标准, 主 要对地理数据的几何空间与整体结构情况进行采集与分析归 纳。此外, 在处理市内地籍调查时由于对精准度的要求较高, 因此, 要采用解析法对数据进行采集。(2)除了解析法与图解 
表 1 GIS 数据属性结构

\begin{tabular}{c|c|c|c|c|c|c|c}
\hline 序号 & 字段名称 & 字段代码 & 字段类型 & 字段长度 & 小数位数 & 值域 & 约束条件 \\
\hline 1 & 标识码 & BSM & Int & 10 & & $>0$ & M \\
\hline 2 & 要素代码 & YSDM & Char & 10 & & & M \\
\hline 3 & 地籍号 & DJH & Char & 19 & & 非空 & O \\
\hline 4 & 使用权类型 & SYQLB & Char & 2 & & & 0 \\
\hline 5 & 土地用途 & TDYT & Char & 4 & & 非空 & M \\
\hline 6 & 规划用途 & GHYT & Char & 4 & & 非空 & O \\
\hline 7 & 实测面积 & SCMJ & Float & 16 & 2 & $>0$ & M \\
\hline 8 & 发证面积 & FZMJ & Float & 16 & 2 & $>0$ & 0 \\
\hline
\end{tabular}

注:约束条件取值: M(必填)、O(选填)。

法, 在对原始数据进行采集时还要明确数据采集对象属性, 也 就是数据信息属性特征与空间结构的关系, 以此为 GIS 数据 建库奠定基础。

\section{3 数据的整理及检验}

在整理数据时可以在专业应用程序上进行，根据项目实 际情况与数据库信息选择合适的处理平台, 以此来提升数据 库整理工作的效率与准确程度。以河北省地矿局第六地质大 队承接项目为例, 在针对不动产类型的工程项目进行数据整 理时通常选用 ArcGIS 软件, 而针对规划性工程的数据信息进 行整理时通常选用 MapGIS 软件。在数据信息整理后针对坐 标定位与属性的准确性进行检验，同时还要保证数据信息的 逻辑性 ${ }^{[3]}$

\section{4 数据入库}

将整理检验后的数据信息导入数据库，是 GIS 数据建库 数据库的建设。

\section{参考文献} 116-121.
的最后环节, 同时也是较为重要的一环。 在此环节中要解决不同平台之间地理数 据的集成难题，也就是要强化数据库中 地质信息的融合程度，当前可以采用数 据转换模式、数据互操作模式以及直接 访问原始数据模式等方式。

\section{4 结语}

综上所述, GIS 数据在建库过程中不仅要保证技术思路 清晰, 还要深入分析工程应用的实际需求, 制定合理的数据库 建库标准。根据测绘工程情况选择合适的数据采集模式,使用 专业软件对数据信息进行整理分析，同时还要检验数据信息 的准确性, 最后通过平台将数据结果导入数据库中, 完成 GIS

[1]谢伟秋,李琳琳,刘振荣.GIS 专题数据采集与建库项目质检研 究[J].测绘与空间地理信息,2016(8):129-132.

[2]史文博.浅谈 MAPSUV 在地籍建库中数据转换的应用 $[\mathrm{J}]$. 黑 龙江科技信息,2016(1):82-83.

[3]李凌.浅谈线变户 GIS 数据采集方法 [J]. 北京测绘,2016(4):

侵人过程中的热变质、蒙脱石化蚀变形成膨润土矿。矿床成因 类型初步确定为火山喷发沉积变质型。

\section{2 找矿标志}

(1)膨润土矿体受流纹质角砾凝灰岩岩层所控制，其成 因与次火山岩体关系密切, 认为沿安山岩及凝灰岩接触带 是找矿的有利部位。(2)地表膨润土矿体露头均属直接找矿 标志。

\section{6 结语}

(1)该区已经找到一个中型膨润土矿, 而且成矿条件优越, 具备形成大型、超大型膨润土矿的条件, 建议对其进一步加大 找矿力度。(2)经过钻探验证发现本区 1 号膨润土矿体东部深 部有变厚趋势, 3 号矿体西端及深部未进行控制,建议下一步 钻探施工应适当进行 1 号矿体 4 线、 6 线以东深部控制以及 3 号矿体西端及深部控制。

\section{参考文献}

[1]辽宁省地质勘查院.中国区域地质志·辽宁志 $[\mathrm{M}]$. 北京:地质出 版社,2017. 